\title{
HIGHER OXIDATIVE STRESS IN CURRENT ALCOHOLICS COMPARED TO ABSTINENT ALCOHOLICS AND NORMAL CONTROLS: A CROSS-SECTIONAL STUDY
}

\author{
Sandhya C. V1, Sreekumari R. G', Vidhukumar $K^{3}$ \\ ${ }^{1}$ Associate Professor, Department of Biochemistry, Government Medical College, Thiruvananthapuram, Kerala. \\ ${ }^{2}$ Assistant Professor, Department of Biochemistry, Government Medical College, Thiruvananthapuram, Kerala. \\ ${ }^{3}$ Additional Professor, Department of Psychiatry, Government Medical College, Thiruvananthapuram, Kerala.
}

ABSTRACT
BACKGROUND
Oxidative stress is considered as one of the key mechanisms in the pathogenesis of alcohol induced organ damage. In the early
recovery phase after alcohol withdrawal also oxidative damage has been demonstrated. Whether this is due to chronic use of alcohol,
or withdrawal from alcohol in an already dependent individual or both is debatable.

AIM

To measure the indictors of oxidative stress in patients currently dependent on alcohol and after alcohol withdrawal for a month and compare them with non-dependent healthy controls.

\section{METHODS}

Thirty alcohol dependent individuals seeking treatment from a tertiary care de-addiction center were assessed for malondialdehyde (MDA), a product of peroxidation of lipids and an indicator of oxidative damage, along with the enzymatic antioxidant superoxide dismutase (SOD). A repeated assay was done during the withdrawal phase, after abstinence for one month Comparison group were healthy individuals without alcohol dependence. The confounding effect of smoking was assessed and adjusted in the above comparison.

\section{RESULTS}

The currently alcohol dependent individuals showed higher levels of MDA compared to both the withdrawal state and normal healthy controls $(p<0.001)$. Independent analysis showed higher levels of MDA for smokers in all the three study groups. The SOD levels were also significantly higher in the current alcoholic subgroup. On withdrawal of alcohol, the SOD levels decreased and became comparable to normal controls. The MDA levels showed significant positive correlation with SOD and duration of dependence in the alcoholic group but not with the average daily intake of alcohol.

\section{CONCLUSION}

Oxidative stress was found to be higher in current alcoholic state than in the withdrawal state. This suggests that the oxidative damage which has been observed in alcohol withdrawal syndrome is not due to withdrawal as such, but triggered by the previous exposure to alcohol.

\section{KEYWORDS}

Oxidative Stress, Alcohol withdrawal, Alcohol Dependence, MDA, SOD, Smoking, ROS.

HOW TO CITE THIS ARTICLE: Sandhya CV, Sreekumari RG, Vidhukumar K. Higher oxidative stress in current alcoholics compared to abstinent alcoholics and normal controls: a cross-sectional study. J. Evolution Med. Dent. Sci. 2016;5(37):2221-2225, DOI: $10.14260 /$ jemds/2016/516

\section{INTRODUCTION}

Alcoholism continues to be a major public health problem worldwide with recent studies showing an incidence of nearly five percent, which includes both alcohol dependence and abuse.(1) Social indulgence in alcohol is also very common in the community. It is said to be one among the five leading causes of preventable morbidity and mortality.(2)

Financial or Other, Competing Interest: None.

Submission 25-03-2016, Peer Review 20-04-2016,

Acceptance 26-04-2016, Published 07-05-2016.

Corresponding Author:

Dr. Sandhya C. V,

Associate Professor,

Department of Biochemistry,

Government Medical College,

Thiruvananthapuram,

Kerala.

E-mail: drsandhyacv@gmail.com

DOI: $10.14260 /$ jemds $/ 2016 / 516$
Alcohol is a drug having numerous metabolic actions and affecting multiple organ systems. According to the WHO Report (2011), alcohol abuse is responsible for at least 60 major types of systemic diseases.(3) Even though there has been a tremendous growth in understanding alcohol metabolism and its actions, the mechanisms by which alcohol causes cell injury are not completely understood and lacunae still exist in understanding the biochemical processes and pathophysiology of alcohol induced organ damage.

Oxidative stress is found to play a central role in many pathways of alcohol induced organ damage.(4),(5) It is a state characterised by excessive production of Reactive Oxygen Species (ROS) and their inadequate scavenging due to impaired antioxidant defences. Liver is the main organ affected by alcohol and many researchers have ascertained the occurrence of increased oxidative stress in experimentally induced Alcoholic Liver Disease (ALD) on animal models and had studied the effect of many therapeutic agents.(6),(7) 
The role of oxidative stress has also been demonstrated in patients with ALD. ${ }^{(8),(9)}$ as well as in other systemic diseases and cases of organ damage caused by alcohol.(10) However, further investigations need to be carried out to confirm whether this oxidative stress is caused by alcohol as such or secondary to the disease process induced by it. There have not been much clinical studies of oxidative stress in apparently healthy alcoholics after adjusting for the effect of other confounders such as smoking and many of them have produced conflicting results.(11),(12),(13)

Meanwhile, some recent studies have shown increased oxidative stress during the alcohol withdrawal state also.(14),(15),(16) As this was found to correlate with glutamatergic excitotoxicity of the withdrawal state, it was put forth as a possible mechanism mediating the symptoms of alcohol withdrawal.(17) The oxidative stress parameters showed correlation with the severity of withdrawal state.(18) but whether this was due to the persistent effect of pathways earlier triggered by alcohol exposure or due to the withdrawal of alcohol needs to be evaluated further.

Measurement of oxidative damage in the alcohol dependant and abstinent states in comparison with nonalcoholic controls after controlling for other factors causing oxidative stress is expected to provide an understanding of the static and dynamic balance of oxidative stress in alcoholism. Hence the present study aimed to examine the oxidative stress parameters in otherwise healthy alcohol dependent individuals in the current or active alcoholic state as well as after a period of abstinence and compare those with normal healthy age matched controls after eliminating the confounding effect of smoking. ROS can react with and cause oxidative damage to complex biomolecules such as lipids (Lipid peroxidation) as well as cause oxidation and depletion of antioxidants involved in free radical scavenging. Thus, oxidative stress can be indirectly assessed by measuring lipid peroxidation, along with assay of antioxidant levels. Lipid peroxidation is the oxidative deterioration of polyunsaturated fatty acids present in the cell membrane leading to formation of lipid hydro peroxides which are subsequently converted to secondary products such as aldehydes and isoprostanes. Malondialdehyde (MDA), which is an important aldehyde product of lipid peroxidation, is used as an indirect index of oxidative damage.(19) in the present study. In order to evaluate the antioxidant status, superoxide dismutase (SOD) which is an important enzymatic antioxidant was selected as the index.

\section{MATERIALS AND METHODS}

The study was conducted in the Deaddiction facility of a tertiary care teaching hospital in South India after obtaining clearance from the Institutional ethics committee according to the provisions of Helsinki declaration. The study group comprised of 30 male alcoholics in the age group 20-50 years without clinical or biochemical evidence of liver disease. Only those patients who were having a valid diagnosis of alcohol dependence as per the DSM-IV TR criteria, duration of dependence more than 5 years and those who had consumed alcohol in the past 24 hours were included in the study. Patients with major systemic illnesses like diabetes, hypertension, coronary artery disease etc., surgical illnesses and comorbid psychiatric disorders were excluded from the study.
Informed consent was obtained for both interview and blood collection. A detailed standardised interview for the collection of data regarding diet, occupation, drinking history, tobacco and drug consumption was performed on all the study subjects. The mean daily alcohol consumption was assessed in terms of standard drink, (Which is defined as $30 \mathrm{ml}$ of hard liquor or half a bottle of beer). Duration of alcoholism was assessed in years. General and systemic examinations were done and blood samples were collected from these patients for evaluation of routine haematological and biochemical parameters, including renal and liver functions tests. Those found to have grossly abnormal results indicating Alcoholic Liver Disease (ALD) or other major systemic illnesses were excluded. This sample was then used for estimation of oxidative stress parameters at baseline. After one month of standard detoxification treatment (Which included benzodiazepines and B-complex vitamins) and abstinence from alcohol, another blood sample was collected for subsequent measurement of oxidative stress.

The control group included 30 healthy male subjects matched for age with alcohol dependent sample. They had no or occasional consumption of alcohol ( $<3$ times a month). Those having significant systemic illnesses and history of regular medication were excluded. Smokers were present in both alcoholic and control groups.

\section{Collection of Samples}

$10 \mathrm{ml}$ of venous blood was collected into plain and EDTA vacutainers. Plasma was separated and used for MDA estimation and lysate prepared from RBC's for Superoxide dismutase assay. Serum which was used for estimation of base line parameters. All the chemicals used for reagent preparation were of analytical grade and only double distilled deionised water was used.

MDA estimation was done using the method of Jentzsch and Bachmann.(20) This method uses high concentration of the synthetic antioxidant Butylated Hydroxy Toluene (BHT) to prevent artefactual formation of lipid hydro peroxides during the assay and applies a baseline correction to the absorbance value, to avoid chromophobes other than MDA- TBA condensation product such as sialic acid, interfering with the assay. Also EDTA added during plasma separation prevents interference by metal ions such as iron. Calibrators were prepared using MDA purchased from Sigma Chemical Co. The SOD activity was measured by an improved pyrogallol auto oxidation method.(21) based on the original method of Marklund and Marklund. The readings were taken in a Beckmann AU400 auto analyser and Systronics UV-visible spectrophotometer.

Results were expressed as means and their standard deviations. Statistical analysis was performed using Statistical Package for social science version 17.0 (SPSS). For each continuous variable, normality was checked and appropriate non-parametric test was chosen for analysis of variables which were not normally distributed. Comparison of mean values of numerical variables between the three different study groups were done using ANOVA for normally distributed and MannWhitney \& rank sum test for non- normally distributed data. $P$ value less than 0.05 considered significant. Comparison of nominal variables were (e.g. smoking) done using Chi-square test. 
Since smoking was found to be a confounding variable which differed significantly among study groups, univariate analysis of variance with smoking as covariate (ANCOVA) was done for the study parameters. Post hoc tests were also done for pair-wise comparison. Spearman's rho rank correlations were done to find the correlations.

\section{RESULTS}

The present study aimed to examine the oxidative stress parameters in otherwise healthy alcohol dependent individuals before and after a period of abstinence (Named as current/active alcoholics and abstinent alcoholics) and compare those with normal healthy age matched controls after eliminating the confounding effect of smoking. The total sample size was 60 comprising of 30 alcoholics and 30 controls. In the alcoholic group, oxidative stress parameters were assayed at the time of presentation (Group 1-Current alcoholics) and also after one month of abstinence from alcohol (Group 2-Abstinent alcoholics). The normal healthy controls were included as Group 3.

Mean ages of subjects in the different study groups were $39.1+8.9$ years for alcoholics and $38.6+10.3$ years for controls and the difference observed was not statistically significant. Measurement of baseline biochemical parameters such as blood sugar, urea, creatinine, uric acid, cholesterol and liver function tests were done on all the subjects. Among these, only AST and uric acid levels showed significant difference between alcoholic and control groups. AST values ranged from 25.1 to $52.3 \mathrm{IU}$ with a mean of $38.7 \mathrm{IU}$ for the alcoholic group and from 17.4 to $26.8 \mathrm{IU}$ with a mean of $22.1 \mathrm{IU}$ for the control group. Analysis showed significant elevation of AST in alcoholics compared to controls $(\mathrm{P}<0.05)$. The difference in ALT and alkaline phosphatase enzyme levels were not significant. AST/ALT ratio (De-Ritis ratio) was increased to over one but below two in the alcoholic groups. The increase in AST preferentially can be explained as due to the rise in the mitochondrial fraction of the enzyme seen in alcoholics. In alcoholic liver disease De-Ritis ratio will be more than two \& thus we can exclude the presence of liver disease in our subjects. The mean value of uric acid was $6.5 \mathrm{mg} / \mathrm{dl}$ in the alcoholic group and $5.2 \mathrm{mg} / \mathrm{dl}$ in the control group. All the other tests showed comparable results between alcoholic and control groups.

The mean duration of dependence in the alcoholic subjects was $7.43+4.3$ years and average amount of alcohol intake was $8.5+5.4($ Mean+S.D) units. Taking the study subjects as a whole, $42 \%$ were non-smokers and $58 \%$ smokers.
The incidence of smoking in the individual groups was 8 non-smokers and 22 smokers in the current alcoholic group, \& 20 non-smokers and 10 smokers in the control group. During detoxification treatment, two of the patients stopped smoking too giving an incidence of 10 non-smokers and 20 smokers in the abstinent alcoholic group. Analysis showed significant difference in the incidence of smoking between groups. The confounding effect of smoking on the parameters of oxidative stress was eliminated by appropriate statistical methods (ANCOVA) and adjusted means calculated. Comparison between groups was made on the basis of this for each parameter studied.

We got higher levels of MDA both in active alcoholics and abstinent alcoholics compared to controls. Univariate analysis of variance for MDA between groups with smoking as covariate (ANCOVA) showed that in addition to the alcohol consumption status of the individual; smoking also affects the MDA levels significantly. Adjusted means were calculated excluding the effect of smoking and differences in the three study groups were tested. MDA levels differed significantly among the three groups (Table 1). Post hoc tests for pairwise comparisons between individual groups also showed MDA significantly elevated in the current or active alcoholics compared to both abstinent alcoholics and controls (Mean difference 178.9 and 224.7 respectively, $\mathrm{P}<0.001)$. Also there was a significant difference in MDA levels between the abstinent alcoholic group and normal healthy controls (Mean difference 45.78, $\mathrm{P}<0.05$ ). Highly significant positive correlation was obtained for MDA with the antioxidant Superoxide dismutase (Table 2). However, MDA values were not found to correlate with the duration of dependence of alcoholism

The mean values obtained for erythrocyte SOD activity in the three different study groups were as shown in table 1 . Increased SOD levels were observed for the active alcoholic group compared to controls, but not for the abstinent group. Analysis was done with smoking as covariate and adjusted means were calculated. The SOD levels were significantly higher in the active alcoholic subgroup compared to controls. Post hoc comparisons also showed significantly increased levels of SOD in the active alcoholic group compared to both abstinent alcoholics and control. (Mean difference 2.93 and 3.18 respectively, $\mathrm{P}<0.001$ ) However, the difference between abstinent alcoholic group and control group is found to be not significant. The SOD levels were found to correlate significantly with MDA levels and also with the average daily amount of alcohol consumption (Table 2).

\begin{tabular}{|c|c|c|c|c|c|c|}
\hline \multirow[b]{2}{*}{ Group } & \multicolumn{3}{|c|}{ MDA (Nanomoles/L) } & \multicolumn{3}{|c|}{ SOD (IU/L) } \\
\hline & $\begin{array}{l}\text { Mean and 95\% Confidence } \\
\text { Interval }\end{array}$ & F value & $P$ value & $\begin{array}{l}\text { Mean and 95\% Confidence } \\
\text { Interval }\end{array}$ & F value & $P$ value \\
\hline Active Alcoholics & $485.2(457.2-513.2)$ & \multirow{3}{*}{70.09} & \multirow{3}{*}{$<0.001$} & $15.96(15.2-16.7)$ & \multirow{3}{*}{21.2} & \multirow{3}{*}{$<0.001$} \\
\hline Abstinent Alcoholics & $306.3(278.6-333.9)$ & & & $13.04(12.0-14.0)$ & & \\
\hline Controls & $260.5(231.7-289.2)$ & & & $12.79(12.0-13.5)$ & & \\
\hline
\end{tabular}

\begin{tabular}{|c|c|c|c|c|c|c|c|c|}
\hline & \multicolumn{2}{|c|}{$\begin{array}{c}\text { Duration of } \\
\text { Dependence }\end{array}$} & \multicolumn{2}{|c|}{ Average Daily Intake } & \multicolumn{2}{c|}{ SOD } & \multicolumn{2}{c|}{ MDA value } \\
\hline & $\mathrm{r}_{\mathrm{s}}$ value & $\mathrm{P}$ value & $\mathrm{r}_{\mathrm{s}}$ value & $\mathrm{P}$ value & $\mathrm{r}_{\mathrm{s}}$ value & P value & $\mathrm{r}_{\mathrm{s}}$ value & - \\
\hline MDA & 0.33 & 0.97 & -0.13 & 0.48 & 0.67 & $<0.001^{* *}$ & - & - \\
\hline SOD & 0.21 & 0.42 & -0.5 & $0.04^{*}$ & - & - & 0.68 & $<0.001^{* *}$ \\
\hline
\end{tabular}

$\mathrm{r}_{\mathrm{s}}$-Spearmann rho value ${ }^{*}$-significant, ${ }^{*}$-highly significant 


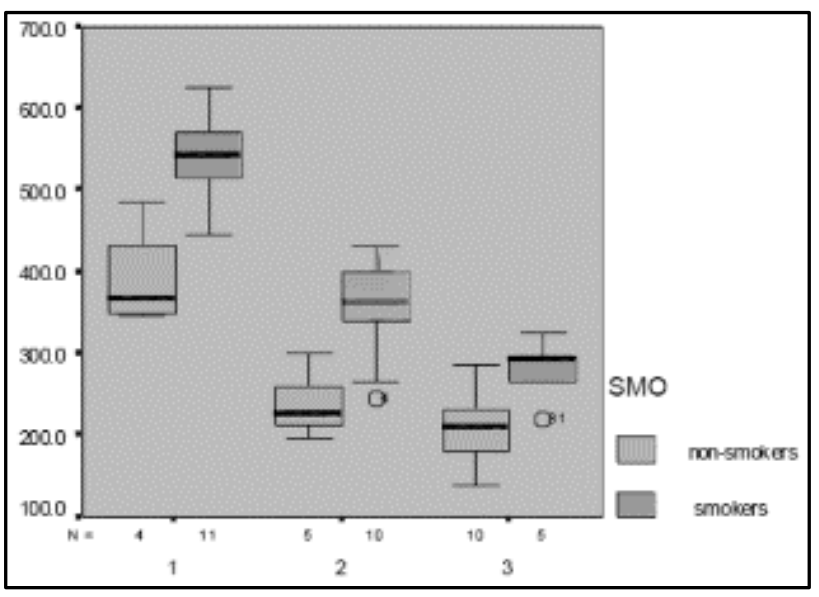

Fig. 1: Box and Whisker's Plot - MDA in different Groups Separately for Smokers and Non-Smokers

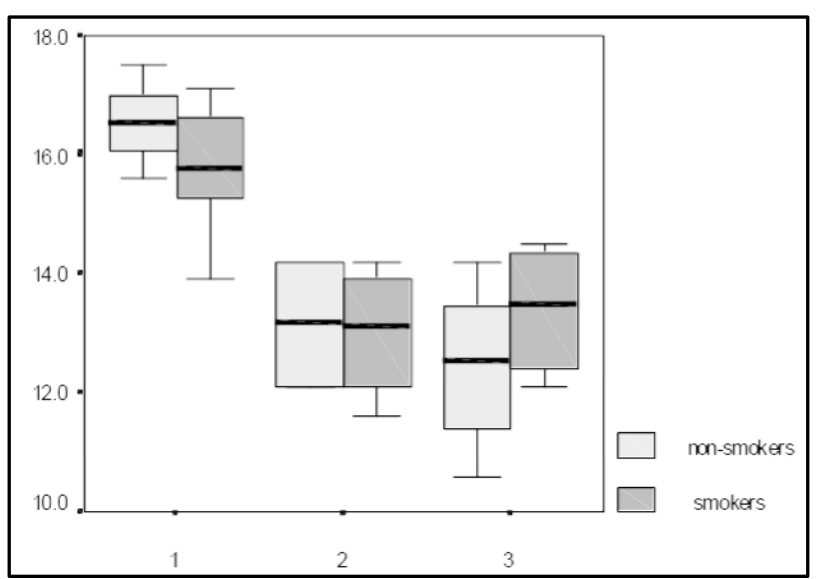

Fig. 2: Box and Whisker's Plot - SOD in different Groups Separately for Smokers and Non-Smokers

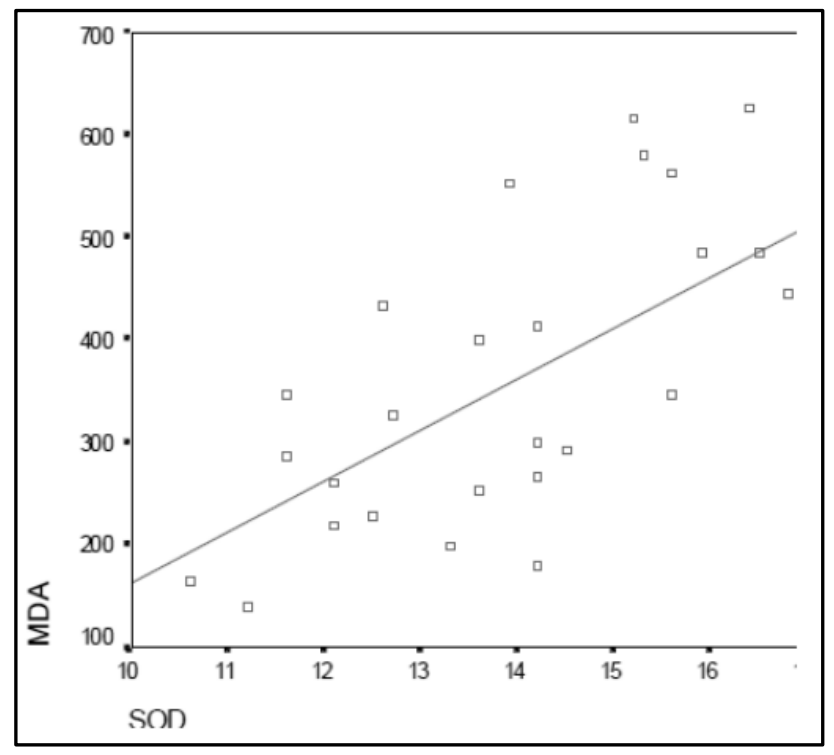

Fig. 3: Correlation of MDA with SOD

\section{DISCUSSION}

The present study demonstrated higher oxidative stress in healthy alcohol dependent individuals without any clinical or biochemical evidence of alcohol induced or other diseases, even after eliminating the confounding effect of smoking.
This shows that oxidative stress is not secondary to any organ damage or systemic illness caused by alcohol, but alcohol itself is the perpetrator of oxidative stress. The prooxidant index MDA was significantly elevated in patients with active alcohol dependence and it decreased significantly on abstinence from alcohol for one month. This clearly shows the higher oxidative stress reported in conditions of alcohol withdrawal is not related to the withdrawal state, but due to the persistent effect of previous alcohol intake leading to increased lipid peroxidation. The mean values obtained for MDA in different groups are comparable to previous studies. In our study a new modified TBARS method was used; which gave results comparable to the HPLC method.(22)

However, the antioxidant SOD was also found to be increased in alcoholics contrary to the expectation that antioxidant levels may decrease due to the oxidative stress. Abstinent alcoholics showed no elevation of the enzyme activity compared to controls and there was a significant difference in the SOD activities between the active and abstinent alcoholics groups. Thus we can see that the higher SOD values in alcoholics tend to fall soon on abstinence and approaches values similar to that of the control group. A possible explanation of this finding is that the SOD levels are increased as a defence mechanism in response to the increased generation of superoxide and other free radicals; in order to scavenge them. Bogdanska J and Todorova B measured SOD activity in the erythrocytes of 37 male patients between 18-59 years with alcohol dependence syndrome without severe liver disease, and found statistically significant increased levels in alcoholics.(23) Other investigators also observed similar findings in red blood cells. (16) Since RBC's mainly contain Cu$\mathrm{Zn} \mathrm{SOD,} \mathrm{we} \mathrm{can} \mathrm{assume} \mathrm{that} \mathrm{Cu}-\mathrm{Zn}$ SOD is elevated in alcoholics. It is however confirmed by various studies that MnSOD levels are also increased in alcoholics the mechanism of this being increased transcription at the genetic level.(24)

However, many studies have also shown decreased levels of SOD in blood cells of alcoholic.(13),(25) This discrepancy between various studies may be due to the different methods used for estimation of SOD and needs further clarification. The auto oxidation assays of SOD have many limitations giving varied levels even in normal subjects.

\section{CONCLUSIONS}

We can assume that alcohol induces oxidative stress which may be responsible for the various organ damage caused by it and this is temporally related to the current status of alcohol consumption as evidenced by the decreased levels of lipid peroxidation in abstinent alcoholics. This proves that the oxidative stress induced by alcohol is reversible on abstinence. Smoking is also associated with increased oxidative stress. Duration of alcohol dependence and the average amount of alcohol intake per day are not associated significantly with the degree of oxidative stress. Current status of alcohol consumption is more important than the above factors.

\section{REFERENCES}

1. Murthy P, Manjunatha N, Subodh BN, et al. Substance use and addiction research in India. Indian J Psychiatry 2010;52(1):S189-99.

2. World Health Organization. The WHO global strategy to reduce the harmful use of alcohol. Alcohol 2010;46:223. Availablefrom:http://www.ncbi.nlm.nih.gov/pubmed/21 508193. 
3. World Health Organisation. Global status report on alcohol and health. World heal organ 2011;122:1-85.

4. Albano E. Alcohol, oxidative stress and free radical damage. Proc Nutr Soc 2006;65(3):278-90.

5. Das SK, Vasudevan DM. Alcohol-induced oxidative stress. Life Sciences 2007;81(3):177-87.

6. Bourogaa E, Jarraya RM, Nciri R, et al. Protective effects of aqueous extract of hammada scoparia against hepatotoxicity induced by ethanol in the rat. Toxicol Ind Health 2014;30(2):113-22.

7. Faremi TY, Suru SM, Fafunso MA, et al. Hepatoprotective potentials of phyllanthus amarus against ethanol-induced oxidative stress in rats. Food Chem Toxicol 2008;46(8):2658-64.

8. Mottaran E, Stewart SF, Rolla R, et al. Lipid peroxidation contributes to immune reactions associated with alcoholic liver disease. Free Radic Biol Med 2002;32(1):38-45. Available from: http://www.ncbi.nlm.nih.gov/pubmed/11755315.

9. Albano E. Oxidative mechanisms in the pathogenesis of alcoholic liver disease. Molecular Aspects of Medicine 2008;29(1-2):9-16.

10. Gonzalez-Reimers E, Santolaria-Fernandez F, MartinGonzalez MC, et al. Alcoholism: a systemic proinflammatory condition. World Journal of Gastroenterology 2014;20(4):14660-71.

11. Lecomte E, Herbeth B, Pirollet P, et al. Effect of alcohol consumption on blood antioxidant nutrients and oxidative stress indicators. Am J Clin Nutr 1994;60(2):255-61. Available from: http://www.ncbi.nlm.nih.gov/pubmed/8030604.

12. Wozniak B, Musialkiewicz D, Wozniak A, et al. Lack of changes in the concentration of thiobarbituric acidreactive substances (TBARS) and in the activities of erythrocyte antioxidant enzymes in alcohol-dependent patients after detoxification. Med Sci Monit 2008;14(1):32$6 . \quad$ Available from: www.medscimonit.com/abstract/index/idArt/636060.

13. Peng FC, Tang SH, Huang MC, et al. Oxidative status in patients with alcohol dependence: a clinical study in Taiwan. J Toxicol Environ Health A 2005;68(17-18):1497509.

14. Gonzaga NA, Mecawi AS, Antunes-Rodrigues J, et al. Ethanol withdrawal increases oxidative stress and reduces nitric oxide bioavailability in the vasculature of rats. Alcohol 2015;49(1):47-56.
15. Huang MC, Chen $\mathrm{CH}$, Peng FC, et al. Alterations in oxidative stress status during early alcohol withdrawal in alcoholic patients. J Formos Med Assoc 2009;108(7):560-9. Available from: $<$ Go to ISI $>: / / 000268345300007$.

16. Parthasarathy R, Kattimani S, Sridhar MG. Oxidative stress during alcohol withdrawal and its relationship with withdrawal severity. Indian J Psychol Med 2015;37(2):175-80. Available from:

http://www.ncbi.nlm.nih.gov/pmc/articles/PMC441825/

17. Tsai GE, Ragan $P$, Chang R, et al. Increased glutamatergic neurotransmission and oxidative stress after alcohol withdrawal. Am J Psychiatry 1998;155(6):726-32.

18. Huang MC, Chen CC, Peng FC, et al. The correlation between early alcohol withdrawal severity and oxidative stress in patients with alcohol dependence. Prog NeuroPsychopharmacology Biol Psychiatry 2009;33(1):66-9. Available from: http://dx.doi.org/10.1016/j.pnpbp.2008.10.009.

19. Dalle-Donne I, Rossi R, Colombo R, et al. Biomarkers of oxidative damage in human disease. Clinical Chemistry 2006;52(4):601-23.

20. Jentzsch AM, Bachmann H, Furst $P$, et al. Improved analysis of malondialdehyde in human body fluids. Free Radic Biol Med 1996;20(2):251-6. Available from: <Go to ISI>://A1996TN60000010.

21. Li X. Improved pyrogallol autoxidation method: a reliable and cheap superoxide-scavenging assay suitable for all antioxidants. J Agric Food Chem 2012;60(25):6418-24.

22. Lapenna D, Ciofani G, Pierdomenico SD, et al. Reaction conditions affecting the relationship between thiobarbituric acid reactivity and lipid peroxides in human plasma. Free Radic Biol Med 2001;31(3):331-5.

23. Bogdanska J, Todorova B, Labudovic D, et al. Erythrocyte antioxidant enzymes in patients with alcohol dependence syndrome. Bratisl Lek Listy 2005;106(3):107-13.

24. Koch OR, Pani G, Borrello S, et al. Oxidative stress and antioxidant defenses in ethanol-induced cell injury. Molecular Aspects of Medicine 2004;25(1-2):191-8.

25. Zhou JF, Chen P. Studies on the oxidative stress in alcohol abusers in China. Biomed Env Sci 2001;14(3):180-8. 